ARTICLE HISTORY: Received: November 14, 2021 Accepted: January 6, 2022 Published: January 13, 2022

DOI: $10.52013 / 2712$

МОДЕЛЬ РЕЛЬСОВОЙ ЛИНИИ С ДИСКРЕТНО-РАСПРЕДЕЛЕННЫМИ ПАРАМЕТРАМИ

Алиев Р.М., Алиев М.М. «ИНФОРМАЦИОННЫЕ СИСТЕМЫ И ТЕХНОЛОГИИ НА ТРАНСПОРТЕ» ТАШКЕНТСКИЙ ГОСУДАРСТВЕННЫЙ ТРАНСПОРТНЫЙ УНИВЕРСИТЕТ

\title{
MODEL OF A RAIL LINE WITH DISCRETELY DISTRIBUTED PARAMETERS
}

\author{
R.M. Aliev, M.M. Aliev \\ DEPARTMENT «INFORMATION SYSTEMS AND TECHNOLOGIES» \\ TASHKENT STATE TRANSPORT UNIVERSITY, RUZ
}

Аннотация. В статье приводятся схемы замещения адаптивной рельсовой цепи с дискретнораспределенными параметрами и предложена её математическая модель.

Abstract. The article provides equivalent circuits for an adaptive rail circuit with discrete-distributed parameters and offers its mathematical model.

Ключевые слова: автоматика, телемеханика, рельсовые цепи, изолирующий стык, зона контроля.

Key words: automation, telemechanic, rail circuits, insulating joint, control zone

\section{Введение}

Для исследования адаптивных рельсовых цепей (АРЦ) схема замещения рельсовой цепи в нормальном и шунтовом режимах должна быть представлена одной обобщенной схемой [1], с возможностью задания неравномерного изменения сопротивления изоляции вдоль рельсовой линии и перемещение поездов любой длины [2]. Контроль состояния АРЦ может осуществляется с учетом состояния и параметров других рельсовых цепей, поэтому схема замещения должна представлять совокупность ряда рельсовых цепей [3]. Такую совокупность рельсовых цепей назовем контрольной зоной (К3) [4]. В контрольную зону могут входить компактно расположенные рельсовые цепи станции или перегона, которые контролируются совместно [5].

Особенность контроля состояния рельсовых цепей методами АРЦ состоит в том, что одновременно должны учитываться значения напряжений приемных концов и токов питающих концов одной или нескольких рельсовых цепей КЗ, что может быть выполнено с участием ЭВМ по программе, соответствующей способу контроля состояния рельсовой цепи [8-12].

На рис. 1 представлена схема подключения приборов к рельсовым линиям [1], которая содержит рельсовые линии Р $Л_{1}$, РЛ $2, \ldots$, РЛ 6 , эквивалентные генераторы $Г Э_{1}, \Gamma Э_{23}, \Gamma Э_{45}$ и эквивалентные приемники ПЭ $1, Э_{2}, \ldots$,

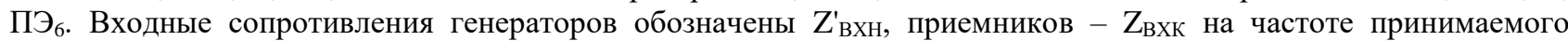
сигнала. Входные сопротивления приемников на других частотах близко к нулю, а генераторов - достаточно большее.

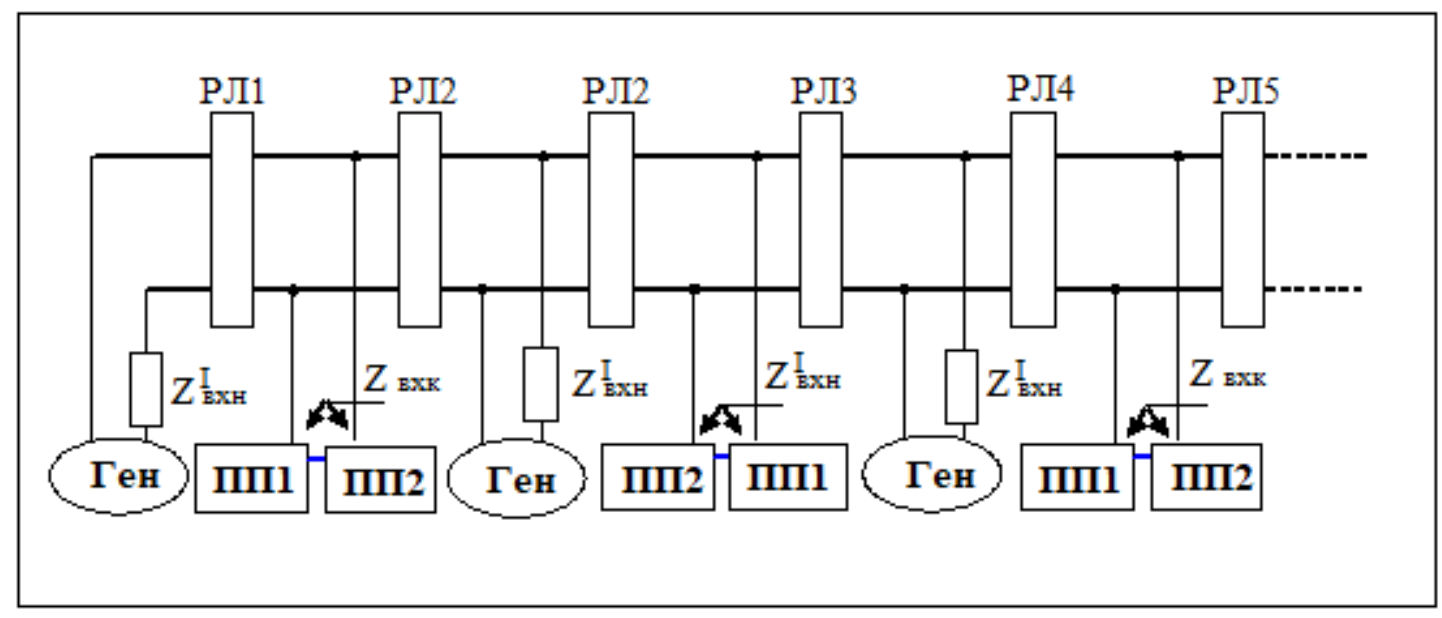




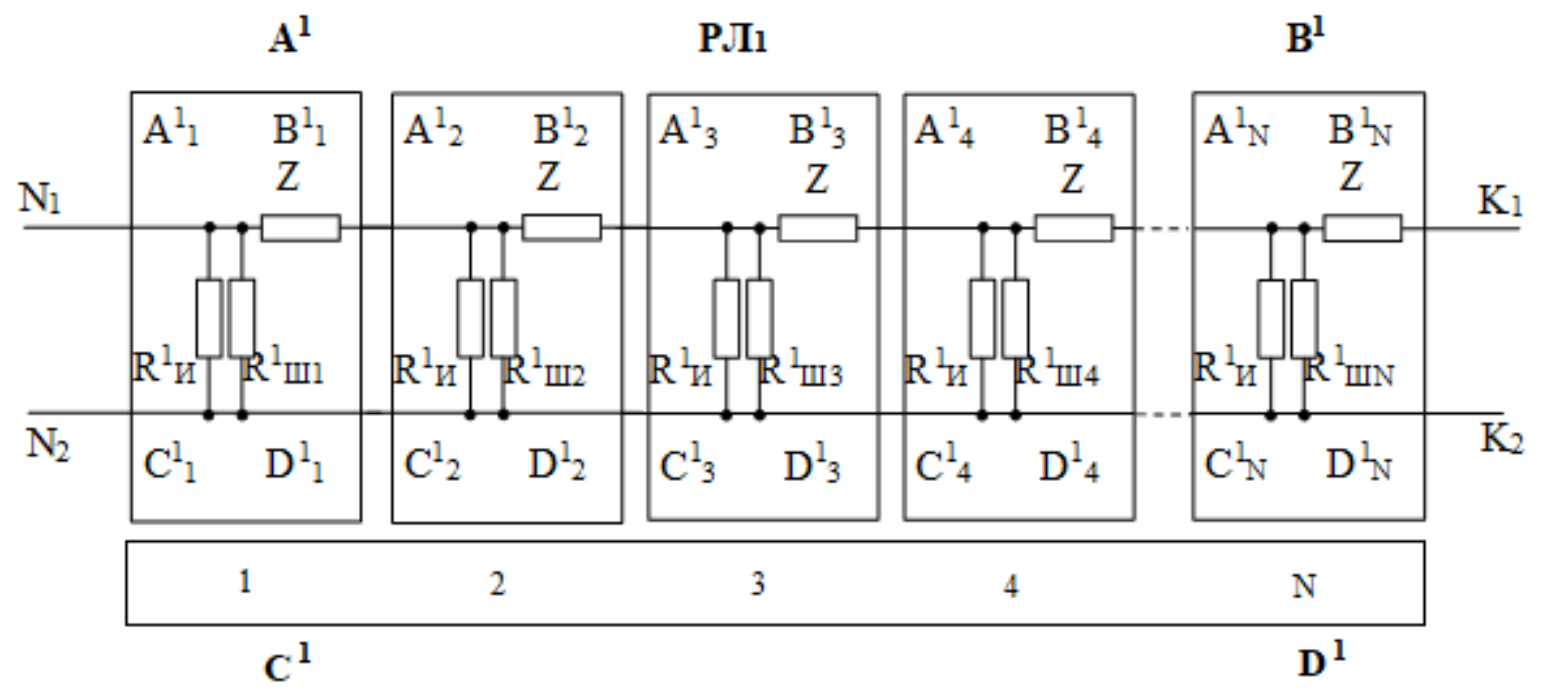

Рисунок 2. Схема замещения рельсовой линии с дискретно-распределенными параметрами

Схема замещения рельсовой линии РЛ 1 изображена на рис. 2. Она содержит $\mathrm{N}$ Г-образных четырехполюсника, в продольные плечи которых включены резисторы с имитирующими сопротивлениями рельс $\mathrm{Z}$, в поперечные плечи параллельно включены резисторы, имитирующие сопротивление изоляции $\mathrm{R}_{\text {и }}$ и

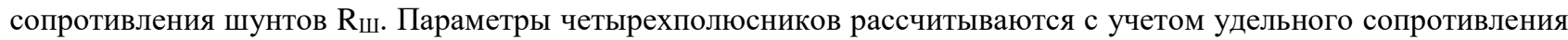
рельс $z$ и изоляции $r_{u}$ при длине участка рельсовой линии 20 м, что соответствует длине условной подвижной единицы.

Коэффициенты $\mathrm{A}^{1}{ }_{1}, \mathrm{~B}^{1}{ }_{1}, \ldots, \mathrm{C}^{6}{ }_{\mathrm{N}}, \mathrm{D}^{6}{ }_{\mathrm{N}}$ рельсовой линии РЛ $_{1}$ имеют верхний и нижний индексы. Верхний индекс указывает на номер четырехполюсника рельсовой линии по рис. 1 , нижний - на номер четырехполюсника участка рельсовой линии по рис. 2.

Схемы замещения рельсовых линий РЛ 2 - РЛ 6 имеют аналогичную структуру.

Математическая модель рельсовой цепи с дискретно-распределёнными параметрами рельсовых линий 


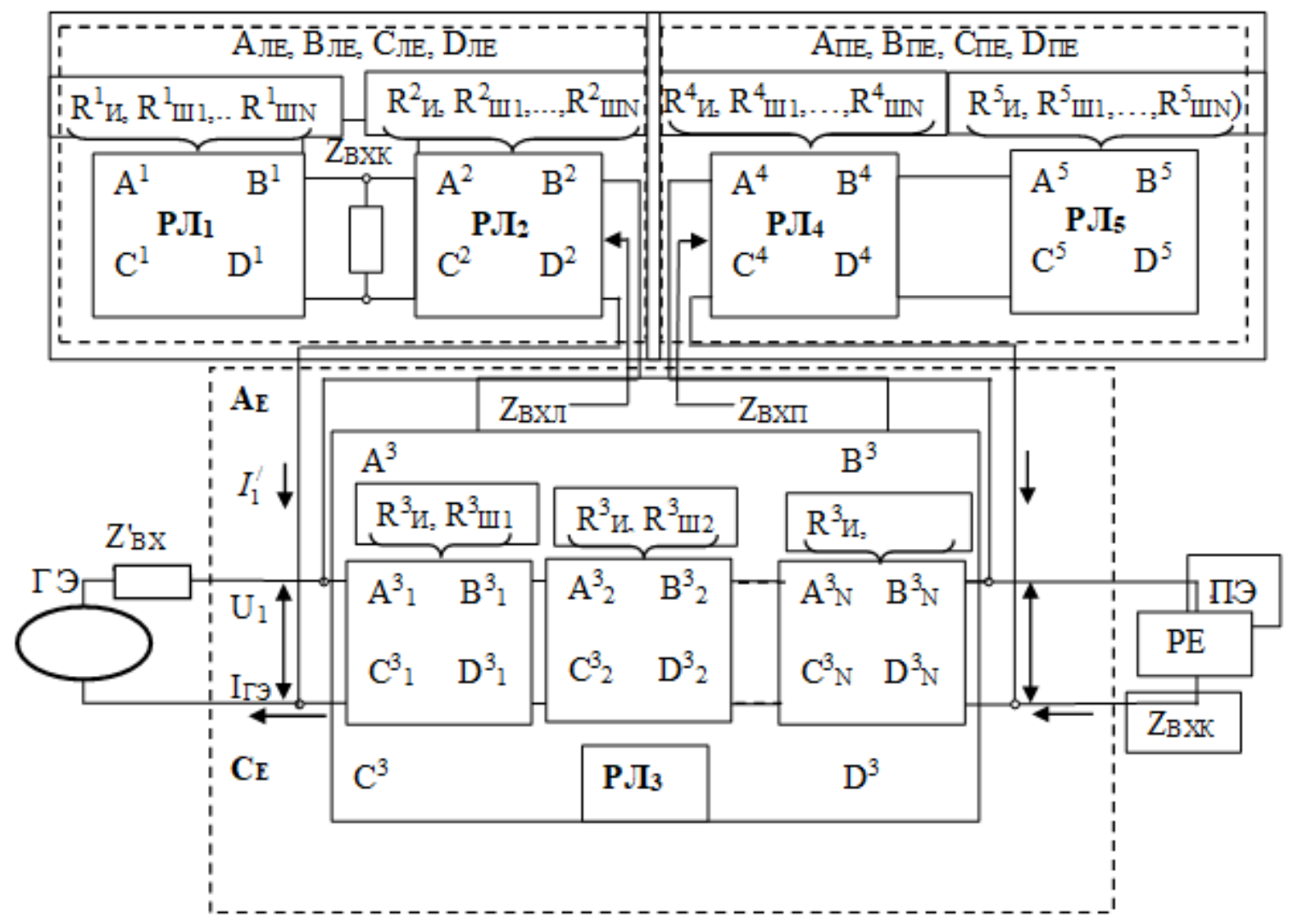

Рисунок 3. Расчётная базовая схема замещения рельсовой цепи

с дискретно-распределёнными параметрами рельсовых линий

Расчет рельсовой цепи должен производиться с учетом влияния других рельсовых линий. При частоте сигнального тока выше 400Гц и длине рельсовых линий 500м и более наблюдается влияние от двух рельсовых линий с каждой стороны от контролируемой рельсовой цепи. Для расчета рельсовой цепи предложена базовая схема замещения рельсовой линии, представленная на рис. 3. На этой схеме основной является рельсовая линия РЛ ${ }_{3}$, а рельсовые линии $\mathrm{P}_{1}, \mathrm{P}_{2}, \mathrm{P}_{4}, \mathrm{P}_{5}$ представлены как влияющие. В базовой схеме используются обозначения коэффициентов четырехполюсников $\mathrm{A}^{1}, \ldots \mathrm{D}^{5}$, сопротивлений изоляции $\mathrm{R}^{1}$ и,.., $\mathrm{R}^{5}$ и и шунтов $\mathrm{R}^{1}$ ш1,... $\mathrm{R}^{5}$ шо в соответствии с рис. 1 и 2.

Для того чтобы установить зависимости между напряжениями и токами по концам рельсовой линии РЛ 3 необходимо определить коэффициенты четырехполюсника $\mathrm{A}^{3}, \mathrm{~B}^{3}, \mathrm{C}^{3}$ и $\mathrm{D}^{3}$ и входные сопротивления $\mathrm{Z}_{\mathrm{BXл}}$ и $\mathrm{Z}_{\mathrm{BXп}}$ смежных рельсовых линий. Коэффициенты $\mathrm{A}^{1}, \mathrm{~B}^{1}, \ldots, \mathrm{C}^{5}, \mathrm{D}^{5}$ определяются по уравнениям:

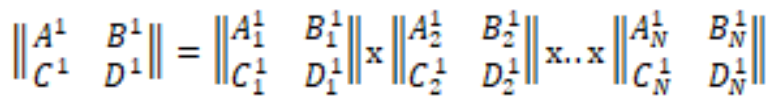

$$
\begin{aligned}
& \left\|\begin{array}{ll}
A^{5} & B^{5} \\
C^{5} & D^{5}
\end{array}\right\|=\left\|\begin{array}{ll}
A_{1}^{5} & B_{1}^{5} \\
C_{1}^{5} & D_{1}^{5}
\end{array}\right\| \mathrm{x}\left\|\begin{array}{ll}
A_{2}^{5} & B_{2}^{5} \\
C_{2}^{5} & D_{2}^{5}
\end{array}\right\|^{\mathrm{x} . . \times \mathrm{x}}\left\|\begin{array}{ll}
A_{N}^{5} & B_{N}^{5} \\
C_{N}^{5} & D_{N}^{5}
\end{array}\right\|
\end{aligned}
$$

Входные сопротивления $Z_{\mathrm{BXл}}$ и $Z_{\mathrm{BXп}}$ определяются через напряжения и токи на входах рельсовых линий РЛ 2 и $\mathrm{P}_{4}:$

$$
Z_{\mathrm{BSI}}=\frac{W_{1}}{I_{1}^{\prime}} ; z_{\mathrm{BXM}}=\frac{U_{2}}{u_{2}^{j}} .
$$


Для определения значений токов $\mathrm{I}_{1}^{\prime}{ }_{1}, \mathrm{I}_{2}^{\prime}$, и напряжений $\mathrm{U}_{1}, \mathrm{U}_{2}$ необходимо найти коэффициенты левого

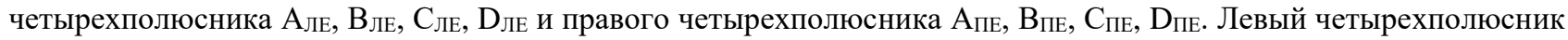
содержит рельсовую линию $\mathrm{P}_{2}$, нагрузку $\mathrm{Z}_{\mathrm{BX \kappa}}$, рельсовую линию $\mathrm{P}_{1}$ Для цепочечного включения упомянутых элементов можно написать:

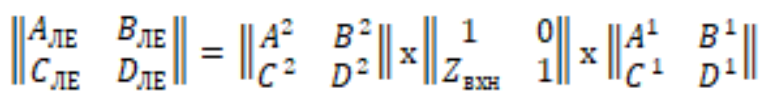

Внешней нагрузки у левого четырехполюсника нет, ток конца отсутствует, и входное сопротивление может быть представлено как

$$
Z_{\mathrm{BXI}}=\frac{A_{\text {IIE }}}{C_{\text {IIE }}} .
$$

Правый эквивалентный четырехполюсник содержит рельсовые линии РЛ 4 и РЛ 5 . Влияние второго путевого генератора, установленного между рельсовыми линиями РЛ 4 и РЛ 5 не учитывается, т.к. его внутреннее сопротивление на частоте первого достаточно большее. Для цепочечного включения этих элементов можно написать:

$$
\left\|\begin{array}{ll}
A_{\Pi E} & B_{\Pi E} \\
C_{\Pi E} & D_{\Pi E}
\end{array}\right\|=\left\|\begin{array}{ll}
A^{4} & B^{4} \\
C^{4} & D^{4}
\end{array}\right\| \mathrm{x}\left\|\begin{array}{ll}
A^{5} & B^{5} \\
C^{5} & D^{5}
\end{array}\right\|
$$

Внешней нагрузки у правого четырехполюсника нет, ток конца отсутствует, и входное сопротивление может быть представлено как

$$
Z_{\mathrm{BXX}}=\frac{A_{\mathrm{IIE}}}{G_{\mathrm{IIE}}}
$$

Коэффициенты эквивалентного четырехполюсника $\mathrm{A}_{\mathrm{E}}, \mathrm{B}_{\mathrm{E}}, \mathrm{C}_{\mathrm{E}}$ и $\mathrm{D}_{\mathrm{E}}$, которые учитывают влияние рельсовых линии и приборов находятся из выражений:

$$
\begin{aligned}
& A_{E}=A^{a}+{ }^{B} / Z_{\text {BXn }} ; \\
& B_{E}=B^{\mathrm{a}} \text {; } \\
& C_{E}=C^{a}+A^{a} / z_{\mathrm{BXI}}+\frac{\mathbb{D}^{3}+B^{3} z_{z_{\max }}}{z_{\mathrm{IxNI}}} ; \\
& D_{E}=D^{a}+B^{a} / Z_{\operatorname{mxn}} .
\end{aligned}
$$

\section{Вывод:}

Расчет напряжений и токов концов рельсовой линии РЛ выполнен при любом сопротивлении изоляции на каждой из рельсовых линий, любых координатах поездов, и любых длинах этих поездов.

\section{Литература}

1. Aliev R. M., Aliev M. M. Method determination of the sensors control of condition track section with an adaptive receiver //Кронос. - 2021. - №. 8 (58). - С. 41-42. 
2. Aliev R. Analysis of controling the state of track sections on lines with speed and high-speed train traffic //Deutsche Internationale Zeitschrift für zeitgenössische Wissenschaft. - 2021. - №. 14. - C. 57-58.

3. Алиев Р. М. Концепция разработки бесстыковых рельсовых цепей //Интерактивная наука. - 2021. - №. 6. - C. 56-57.

4. Aliev R., Aliev M., Tokhirov E. Model microprocessor device of four-wire scheme of the direction change //Deutsche Internationale Zeitschrift für zeitgenössische Wissenschaft. - 2021. - №. 11-1. - C. 30-32.

5. Aliev R. Analysis of the track sections control system a rolling stock axle counting sensor //AIP Conference Proceedings. - AIP Publishing LLC, 2021. - T. 2439. - №. 1. - C. 020015.

6. Aliev R. M., Aliev M. M. Mathematical model of the sensor for controling the condition of the track section with an adaptive receiver at the free condition of the controlled section //Journal of Physics: Conference Series. - IOP Publishing, 2021. - T. 1973. - №. 1. - С. 012021.

7. Полевой Ю.И. Совершенствование устройств контроля состояния железнодорожных путевых участков. Самара, 2005, стр.133.

8. Аркатов В.С., Кравцов Ю.А., Степенский Б.М. Рельсовые цепи:Анализ работы и техническое обслуживание. М.,-Транспорт,1990, стр.295.

9. Дмитриев В.С., Минин В.А. Системы автоблокировки с рельсовыми цепями тональной частоты. М.,Транспорт,1992.,стр.184

10. Theeg G. \& Vlasenko S. 2020 Railway Signalling\& Interlocking. 3ed Edition. Germany, Leverkusen, PMC Media House GmbH, - 2020. 552 p.

11. Sedykh D., Gordon M., Zuyev D. \&Skorokhodov A. 2018 Analysis of the Amplitude and Phase-Manipulated Signals of Automation Devices via Bluetooth Technology. Proceedings of 16th IEEE East-West

12. Design \& Test Symposium (EWDTS 2018). Russia, Kazan, pp. 703-710. DOI: 10.1109/EWDTS.2018.8524605.

13. Efanov D. V., Osadchy G. V., Khóroshev V. V. \&Shestovitskiy D. A. 2019 Diagnosticsof Audio-Frequency Track Circuits in Continuous Monitoring Systems for Remote ControlDevices: Some Aspects. Proceedings of 17th IEEE East-West Design \& Test Symposium (EWDTS 2019). Batumi, Georgia, pp. 162-170. DOI: 10.1109/EWDTS.2019.8884416.

\section{References}

1. Aliev R. M., Aliev M. M. Method determination of the sensors control of condition track section with an adaptive receiver //Кронос. - 2021. - №. 8 (58). - С. 41-42.

2. Aliev R. Analysis of controling the state of track sections on lines with speed and high-speed train traffic //Deutsche Internationale Zeitschrift für zeitgenössische Wissenschaft. - 2021. - №. 14. - C. 57-58.

3. Алиев Р. М. Концепция разработки бесстыковых рельсовых цепей //Интерактивная наука. - 2021. - №. 6. - C. 56-57.

4. Aliev R., Aliev M., Tokhirov E. Model microprocessor device of four-wire scheme of the direction change //Deutsche Internationale Zeitschrift für zeitgenössische Wissenschaft. - 2021. - №. 11-1. - C. 30-32.

5. Aliev R. Analysis of the track sections control system a rolling stock axle counting sensor //AIP Conference Proceedings. - AIP Publishing LLC, 2021. - T. 2439. - №. 1. - C. 020015.

6. Aliev R. M., Aliev M. M. Mathematical model of the sensor for controling the condition of the track section with an adaptive receiver at the free condition of the controlled section //Journal of Physics: Conference Series. - IOP Publishing, 2021. - T. 1973. - №. 1. - C. 012021.

7. Polevoy Yu.I. Improvement of devices for monitoring the state of railway track sections. Samara, 2005, p. 133.

8. Arkatov V.S., Kravtsov Yu.A., Stepensky B.M. Track circuits: Performance analysis and maintenance. M., Transport, 1990, p. 295.

9. Dmitriev V.S., Minin V.A. Auto-blocking systems with voice frequency track circuits. - M., Transport, 1992. , p. 184 Theeg G. \& Vlasenko S. 2020 Railway Signalling\& Interlocking. 3ed Edition. Germany, Leverkusen, PMC Media House GmbH, - 2020. 552 p.

10. Sedykh D., Gordon M., Zuyev D. \&Skorokhodov A. 2018 Analysis of the Amplitude and Phase-Manipulated Signals of Automation Devices via Bluetooth Technology. Proceedings of 16th IEEE East-West

11. Design \& Test Symposium (EWDTS 2018). Russia, Kazan, pp. 703-710. DOI: 10.1109/EWDTS.2018.8524605.

12. Efanov D. V., Osadchy G. V., Khóroshev V. V. \&Shestovitskiy D. A. 2019 Diagnosticsof Audio-Frequency Track Circuits in Continuous Monitoring Systems for Remote ControlDevices: Some Aspects. Proceedings of 17th IEEE East-West Design \& Test Symposium (EWDTS 2019). Batumi, Georgia, pp. 162-170. DOI: 10.1109/EWDTS.2019.8884416. 